
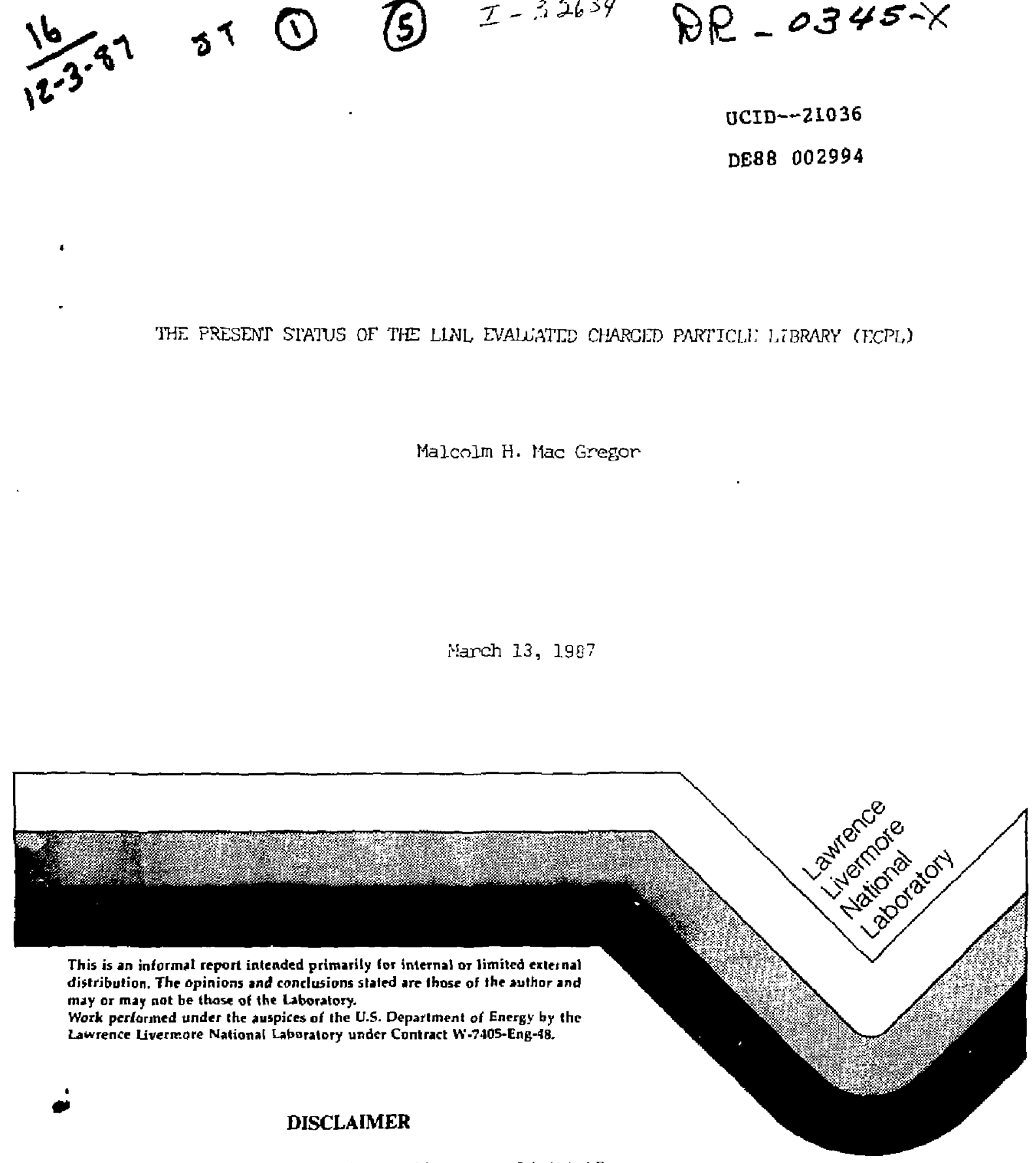

This tepon wis prepared as an account of wotk sponsored by an agency of the Uniced Siates Government. Neithes the United Stales Government nor any agency thereor, nor any of their employes, makes any wartanty, express or implicd, or assumes any tegal liabilily or tesponsibility for the accuracy. completeness, or usefulness of any information. upparatus, product, of process disclosed. or repsesents that its use nould not infringe privately owned rights, Refer. ence herein to any specific commercial producl, process, or service by trade name, Irademark, manufacturet. or utherwise does nor necessarily constitute or imply its endotscment, recommendation, or favoring by the United States Government of any agency thereor. The views MASTER and opinions of authers txpressed berein da nol necessarily stute of reflect those of the and opinions of authors txpressed herein do no 
THE PRESENT STATUS OF THE LUNL EVAUUATED CHIARGED PARTICLE LIBRARY (ECPL)

\author{
Malcoln H. Mac Gregor \\ Lawrence livermore National Laboratory * \\ Livernore, Califomia 94550 \\ March 13, 1987
}

The Nuclear Data Group of the Lawrence Livermore National Laboratory has for the past decade maintained an Evaluated Charged Particle Library (ECPL). This ECPL library encompasses low-Z tanget muclides and low-Z incident and outgoirs projectiles. Each evaluated data set extends from threshoid to $20 \mathrm{MeV}$ incident projectile energy, and it includes a complete set of data: total cross sections, angular distributions, energy distributions of the final-state particles, energy deposits, Maxwellian average reaction rates, and in-flight reaction cross sections. The Nuclear Data Group also maintains a reprint library of experimental papers on low-Z charged-particle reactions.

The present report is written with two purposes in mind: (1) to summarize the charged-particle reactions that presently exist in ECPL (see Table I); and (2) to list all of the low-Z charged-particle reactions for which data exist in the livermone experimental data compilation (cee reactions listed in Table II). The reactions in Table II that are underlined are presently included in ECPL. It is clear that only a small fraction of the reactions listed in Table II have as yet been included in ECPL. However, the reactions that are now in ECPL are the most impontant ones. Also, some of the reaction channels listed in Table II are redundant. Furthermore, although data exist for all of the reactions shown in Table II, some of these data are very fragmentary, and are not sufficient to permit an accurate evaluation to be carried out. In this regard, it should be 
noted that charged particle data, by their very nature, tend in general to be incomplete, since experimentalists are usually more interested in testing nuclear models than in providing complete data sets. Thus the evaluated data sets in ECPL are drawn partly from experimental data, partly from systematics, and partly from computer modeling codes.

This report is intended primarily for users at Livermore, but it may also be of interest to workers outside of the Laboratory who are involved in fusion energy. The ECPL 1 ibrary and its associated processing codes are maintained on the Livermore CRAY computers. These files and codes can be made generally availabie on the MFECC computers to the fusion energy commuity.

Two comprehensive tables are given below which summarize the current status of the Livermore Evaluated Changed Particle Library. The first table lists all of the reactions which are currently included in ECPL. The second table lists all of the reactions for which data exist in the Livermore compilation of charged particle data, with the reactions underlined if they have been entered into ECPL. Also included are two auxiliary tables that contain the definitions of the incident particles $\left(Y_{i}\right)$ and the reaction types ( $C$ numbers), as used in the standand Livermore ECPL notation.

tWork performed under the auspices of the U. S. Department of Energy by the Lawrence Livermore National Labonatory under contract number W-7405-ENG-48, 
Table I. Reactions that are currently included in the ECPL evaluated changed particle library. The coulomb reactions are written as IACS and SI, where LACS denotes Large Angle Coulomb Scattering, and SI denotes Scattering plus Interaction (SI = total elastic scattering - LACS). The nuclear reactions are written in the form $Y_{i}, Y_{o}$ with $Y_{i}$ denoting the incident particle and $Y_{0}$ denoting the cutgoing particle (or particles) of interest.

\begin{tabular}{|c|c|c|c|c|c|}
\hline \multirow{2}{*}{$\begin{array}{l}\text { Target } \\
\text { particle } \\
\text { P }_{P}\end{array}$} & \multirow{2}{*}{$\begin{array}{c}\begin{array}{c}\text { Incident } \\
\text { panticle }\end{array} \\
P\end{array}$} & \multicolumn{2}{|c|}{$\begin{array}{l}\text { Coulonb } \\
\text { interactions }\end{array}$} & $\begin{array}{l}\text { Nuclear } \\
\text { reactions }\end{array}$ & \multirow{2}{*}{$\begin{array}{l}\text { ECPL } \\
\text { C-numbers } \\
8,9\end{array}$} \\
\hline & & LACS & SI & \multirow{5}{*}{$T, n$} & \\
\hline & D & LACS & SI & & 8,9 \\
\hline & $T$ & LACS & SI & & $8,9,11$ \\
\hline & ${ }^{3} \mathrm{He}$ & IACS & SI & & 8,9 \\
\hline & $\alpha$ & LACS & $S I$ & & 8,9 \\
\hline \multirow[t]{5}{*}{$\mathrm{D}$} & $\mathrm{p}$ & IACS & $S I$ & \multirow{4}{*}{$\begin{array}{l}\mathrm{D}, \mathrm{D} \quad \mathrm{D}, \mathrm{P} \\
\mathrm{T}, \mathrm{N} \quad \mathrm{T}, \mathrm{ra} \alpha \\
3_{\mathrm{He}, \mathrm{P}}\end{array}$} & 8,9 \\
\hline & $\mathrm{D}$ & LACS & $S I$ & & $8,9,11,40$ \\
\hline & $T$ & LACS & SI & & $8,9,11,30$ \\
\hline & ${ }^{3} \mathrm{He}$ & LACS & $\mathrm{SI}$ & & $8,9,40$ \\
\hline & $a$ & ¿ACS & $S I$ & $\alpha, n p \quad \alpha, p n$ & $8,9,20,21$ \\
\hline \multirow[t]{5}{*}{$\mathrm{T}$} & p & LACS & SI & \multirow{5}{*}{$\begin{array}{ll}\mathrm{P}, \mathrm{N} & \mathrm{P}, \mathrm{\gamma} \\
\mathrm{D}, \mathrm{n} & \mathrm{D}, \gamma \cap \mathrm{\alpha} \\
\mathrm{T}, 2 \mathrm{n} & \end{array}$} & $8,9,11,46$ \\
\hline & $\mathrm{D}$ & LACS & $\mathrm{SI}$ & & $9,9,11,30$ \\
\hline & $\mathrm{T}$ & IACS & $S I$ & & $8,9,12$ \\
\hline & $3_{\mathrm{He}}$ & LACS & $S I$ & & 8,9 \\
\hline & $a$ & LACS & $S I$ & & 8,9 \\
\hline \multirow[t]{5}{*}{${ }^{3} \mathrm{He}$} & $\mathrm{p}$ & LACS & SI & \multirow{5}{*}{$\begin{array}{l}\mathrm{D}, \mathrm{p} \\
\mathrm{T}, \mathrm{nP} \quad \mathrm{T}, \mathrm{pn} \quad \mathrm{T}, \mathrm{D}\end{array}$} & 8,9 \\
\hline & D & LACS & SI & & $8,9,40$ \\
\hline & $T$ & LACS & $S I$ & & $8,9,20,21,41$ \\
\hline & ${ }^{3} \mathrm{He}$ & LACS & SI & & 8,9 \\
\hline & $\alpha$ & LACS & SI & & 8,9 \\
\hline
\end{tabular}


Table I (continued).

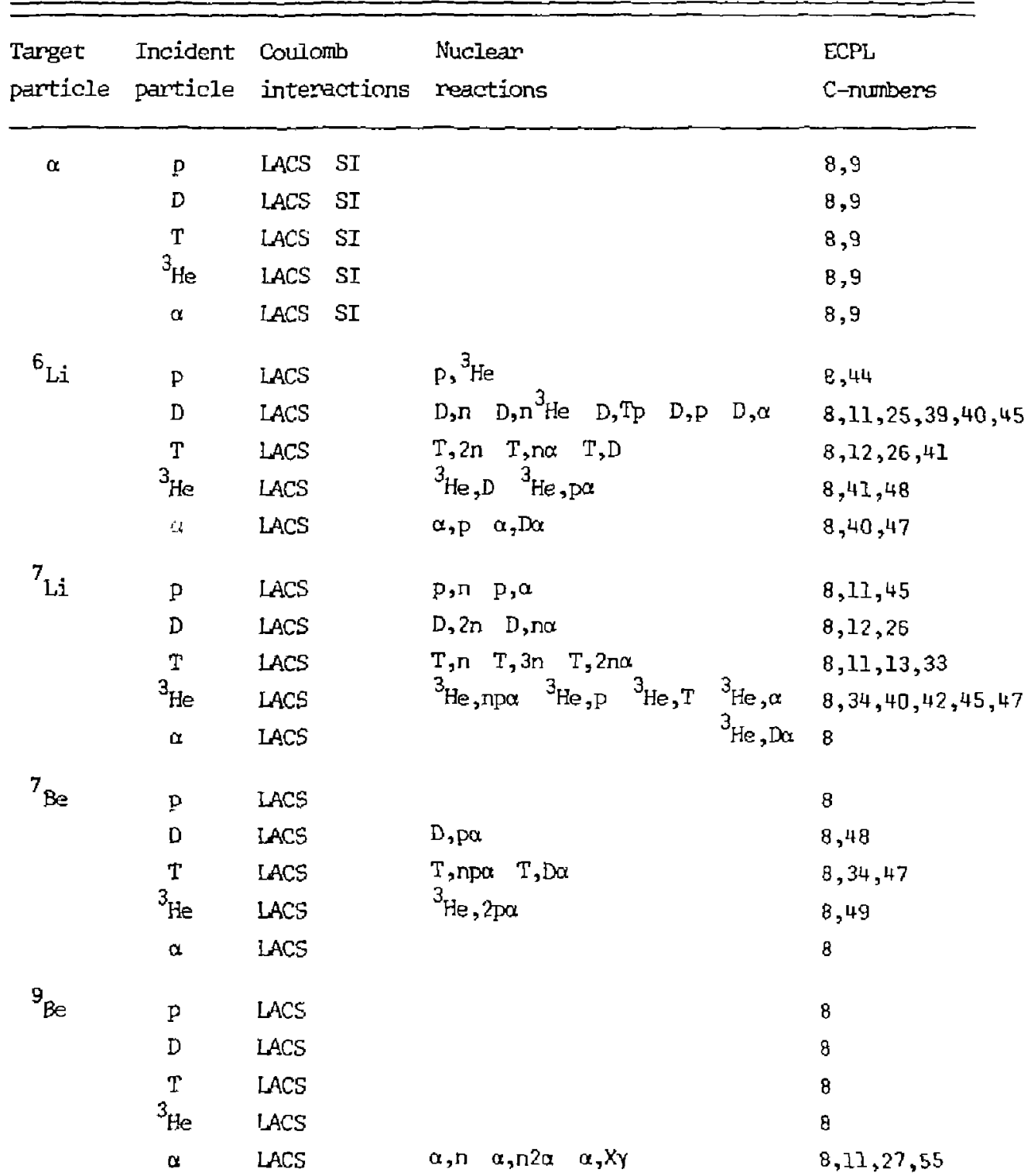


Table I (continued)

\begin{tabular}{|c|c|c|c|c|}
\hline $\begin{array}{l}\text { Target } \\
\text { particle }\end{array}$ & $\begin{array}{l}\text { Incident } \\
\text { particle }\end{array}$ & $\begin{array}{l}\text { Coulomb } \\
\text { interactions }\end{array}$ & $\begin{array}{l}\text { Nuclear } \\
\text { reactions }\end{array}$ & $\begin{array}{l}\text { ECPL } \\
\text { C-numbers }\end{array}$ \\
\hline \multirow[t]{5}{*}{${ }^{10} \mathrm{~B}$} & $\mathrm{P}$ & LACS & $\mathrm{p}, \alpha^{3} \mathrm{He} \quad \mathrm{p}, \alpha$ & $8,38,45$ \\
\hline & $D$ & LACS & $D, n \quad D, 3 \alpha \quad D, P$ & $8,11,37,40$ \\
\hline & $\mathrm{T}$ & LACS & & 8 \\
\hline & ${ }^{3} \mathrm{He}$ & LACS & & 8 \\
\hline & $\alpha$ & LACS & & 8 \\
\hline \multirow[t]{5}{*}{${ }^{11}{ }_{B}$} & $\mathrm{P}$ & LACS & $\mathrm{p}, \mathrm{n} \quad \mathrm{p}, 3 \alpha$ & $8,11,37$ \\
\hline & $D$ & LACS & $D, n \quad D, p$ & $8,11,40$ \\
\hline & $\mathrm{T}$ & LACS & & 8 \\
\hline & ${ }^{3} \mathrm{He}$ & IACS & & 8 \\
\hline & $\alpha$ & LACS & & 8 \\
\hline \multirow[t]{5}{*}{${ }^{12} \mathrm{C}$} & $\mathrm{p}$ & LACS & & 8 \\
\hline & $\mathrm{D}$ & LACS & & $\mathrm{B}$ \\
\hline & $T$ & LACS & & 8 \\
\hline & ${ }^{3} \mathrm{He}$ & LACS & & 8 \\
\hline & $\alpha$ & LACS & & 8 \\
\hline \multirow[t]{5}{*}{$24 \mathrm{~N}$} & $p$ & LACS & & 8 \\
\hline & $D$ & LACS & & 8 \\
\hline & $T$ & LACS & & 8 \\
\hline & ${ }^{3} \mathrm{He}$ & LACS & & $B$ \\
\hline & $\alpha$ & LACS & & 8 \\
\hline \multirow[t]{5}{*}{${ }^{16} 0$} & $p$ & LACS & & 8 \\
\hline & $\mathrm{D}$ & LACS & & 8 \\
\hline & $\mathrm{T}$ & LACS & & 8 \\
\hline & $3_{\mathrm{He}}$ & LACS & & 8 \\
\hline & $\alpha$ & LACS & & 8 \\
\hline${ }^{89} Y$ & $\mathrm{P}$ & & $\mathrm{p}, \mathrm{n}$ & 11 \\
\hline
\end{tabular}


Table II. Charged-particle reactions for which experimental data exist in the Livermore data compilation. Reactions that have been entered into ECPL. are underlined. The $Y_{i}$ and $C$ descriptors are defined in Tables Al and A2.

\begin{tabular}{|c|c|c|}
\hline $\mathrm{ZA}$ & $Y_{i}$ & $\mathrm{c}$ \\
\hline \multirow[t]{3}{*}{1001} & 2 & $8+9,46$ \\
\hline & 3 & 11,20 \\
\hline & 4 & $\underline{B+9}, \underline{11}$ \\
\hline \multirow[t]{5}{*}{1002} & 2 & $8+9,11,21,40,46$ \\
\hline & 3 & $8+9,11,20,40,42,44,46$ \\
\hline & 4 & $\underline{8}+\underline{9}, 45$ \\
\hline & 5 & $\underline{40}, 46$ \\
\hline & 6 & $9+9,11,21,40,45,46$ \\
\hline \multirow[t]{5}{*}{1003} & 2 & $\underline{8}+\underline{9}, \underline{11}, 44,46$ \\
\hline & 3 & $\underline{8}+9,11,12,20,30,40,46$ \\
\hline & 4 & $\underline{8}+\underline{9}, \underline{12}$ \\
\hline & 5 & $\underline{8}+9,11,40,41,46$ \\
\hline & 6 & 11,46 \\
\hline \multirow[t]{5}{*}{2003} & 2 & $\underline{9}+\underline{9}$ \\
\hline & 3 & $\underline{8}+\underline{9}, I], 40,45,46$ \\
\hline & 4 & $8+9,11,40,41$ \\
\hline & 5 & $9+9,40,45,46$ \\
\hline & 6 & 40,46 \\
\hline \multirow[t]{5}{*}{2004} & 2 & $9+9,20,41,42,46$ \\
\hline & 3 & $8+9,11,20,40,41$ \\
\hline & 4 & $\underline{8}+9,40$ \\
\hline & 5 & $\underline{8}+\underline{9}, 40,45,46$ \\
\hline & 6 & $\underline{B}+\underline{9}$ \\
\hline
\end{tabular}


Table II (continued).

\begin{tabular}{|c|c|c|}
\hline $\mathrm{ZA}$ & $Y_{i}$ & $\mathrm{c}$ \\
\hline \multirow[t]{5}{*}{3006} & 2 & $9+9,11,20,40,41,42,44,45,46$ \\
\hline & 3 & $\underline{8}+9,11,25,39,40,41,42,44,45,46$ \\
\hline & 4 & $11,12,40,41$ \\
\hline & 5 & $8+9,11,40,41,42,43,44,45$ \\
\hline & 6 & $\underline{8}+9,11,4 \underline{40}, 41,42,45,46$ \\
\hline \multirow[t]{5}{*}{3007} & 2 & $\underline{8}+9, \underline{11}, 25,40,41,42, \underline{45}, 46,55$ \\
\hline & 3 & $8+9,11,12,40,4 i, 42,45,46$ \\
\hline & 4 & $11,12,40,45,46$ \\
\hline & 5 & $8+9,11,21,41,41,42,44,45,46,55$ \\
\hline & 5 & $8+9,11,40,42,45,46$ \\
\hline \multirow[t]{2}{*}{4007} & 2 & 45 \\
\hline & 3 & 40 \\
\hline \multirow[t]{5}{*}{4009} & 2 & $\underline{8}+9,11,12,21,40,41,42,45,46,55$ \\
\hline & 3 & $8+9,11,40,41,42,45,46,55$ \\
\hline & 4 & $8+9,11,40,41,45$ \\
\hline & 5 & $\underline{8}+9,11,12,37,40,41,42,45,46,55$ \\
\hline & 6 & $8+9, \underline{11}, 12,41,45, \underline{55}$ \\
\hline \multirow[t]{2}{*}{4010} & 2 & $8+9,40,46$ \\
\hline & 3 & $8+9,40,42,45,46$ \\
\hline \multirow[t]{5}{*}{5010} & 2 & $\underline{8}+9,11,40,41,44,45,46,55$ \\
\hline & 3 & $\underline{8}+9, \underline{11}, \underline{37}, \underline{40}, 41,42,44,45$ \\
\hline & 4 & $8+9,40,41,45$ \\
\hline & 5 & $\underline{8}+9,11,40,41,42,44,45,46$ \\
\hline & 6 & $8+9,11,40,41,42,45,55$ \\
\hline
\end{tabular}


Table II (continued).

\begin{tabular}{|c|c|c|}
\hline $\mathrm{ZA}$ & $Y_{i}$ & $\mathrm{C}$ \\
\hline \multirow[t]{5}{*}{5011} & 2 & $\underline{8}+9, \underline{11}, 26, \underline{37}, 40,41,45,116$ \\
\hline & 3 & $8+9,11,12,27, \underline{40}, 42,45,46$ \\
\hline & 4 & $8+9,40,45$ \\
\hline & 5 & $\underline{8}+9,11,40,41,42,45,46$ \\
\hline & 6 & $\underline{8}+9,11,40,41,42,46,55$ \\
\hline \multirow[t]{5}{*}{6012} & 2 & $\underline{8}+9,45,46,55$ \\
\hline & 3 & $8+9,11,40,41,42,45$ \\
\hline & 4 & $\underline{8}+9,40,41,45$ \\
\hline & 5 & $8+9,11,40,41,42,44,45$ \\
\hline & 6 & $8+9,11,40,45,46$ \\
\hline \multirow[t]{5}{*}{6013} & 2 & 11,46 \\
\hline & 3 & $8+9,11,40,42,45,46$ \\
\hline & 4 & 41 \\
\hline & 5 & $8+9,11,40,42,45$ \\
\hline & 6 & $11,42,55$ \\
\hline \multirow[t]{3}{*}{6014} & 2 & 41,42 \\
\hline & 3 & 40,45 \\
\hline & 5 & $8+9$ \\
\hline \multirow[t]{4}{*}{7014} & 2 & $\underline{8}+9,45,46,55$ \\
\hline & 3 & $8+9,40,41,45,46$ \\
\hline & 5 & $\underline{8}+9,41,45$ \\
\hline & 6 & 40 \\
\hline \multirow[t]{3}{*}{7015} & 2 & 11,45 \\
\hline & 3 & 11 \\
\hline & 5 & 11 \\
\hline
\end{tabular}


Table II (continued).

\begin{tabular}{|c|c|c|}
\hline $\mathrm{ZA}$ & $Y_{i}$ & $\mathrm{C}$ \\
\hline \multirow[t]{5}{*}{8016} & 2 & $8+9,40,41,42,45,55$ \\
\hline & 3 & $8+9,11,40,41,45$ \\
\hline & 4 & 40,45 \\
\hline & 5 & $8+9,40,45$ \\
\hline & 6 & $8+9$ \\
\hline 8017 & 2 & 45 \\
\hline \multirow[t]{3}{*}{8018} & 2 & $11,41,42,45$ \\
\hline & 3 & 40 \\
\hline & 5 & 11 \\
\hline \multirow[t]{3}{*}{9019} & 2 & $8+9,11,40,45,46$ \\
\hline & 3 & $3+9,11$ \\
\hline & 5 & $8+9,11,44,45$ \\
\hline 10022 & 5 & 11 \\
\hline \multirow[t]{3}{*}{11023} & 2 & $8+9$ \\
\hline & 5 & $8+9$ \\
\hline & 6 & $8+9,13$ \\
\hline \multirow[t]{2}{*}{12024} & 3 & 41. \\
\hline & 5 & 12 \\
\hline \multirow[t]{3}{*}{13027} & 2 & $8+9,11,46$ \\
\hline & 3 & 41 \\
\hline & 5 & $12,44,45$ \\
\hline 15031 & 2 & $8+9,40$ \\
\hline 17035 & 5 & $8+9$ \\
\hline
\end{tabular}


Table Al. Definitions of $Y_{i}$ particle descriptors.

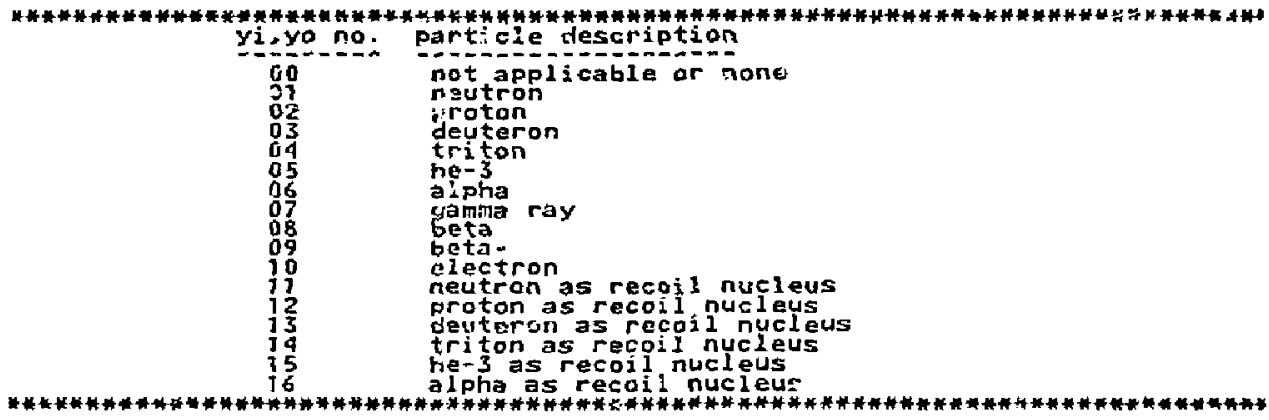

Tabie A2. Detinitions of $C$ reaction descriptors.

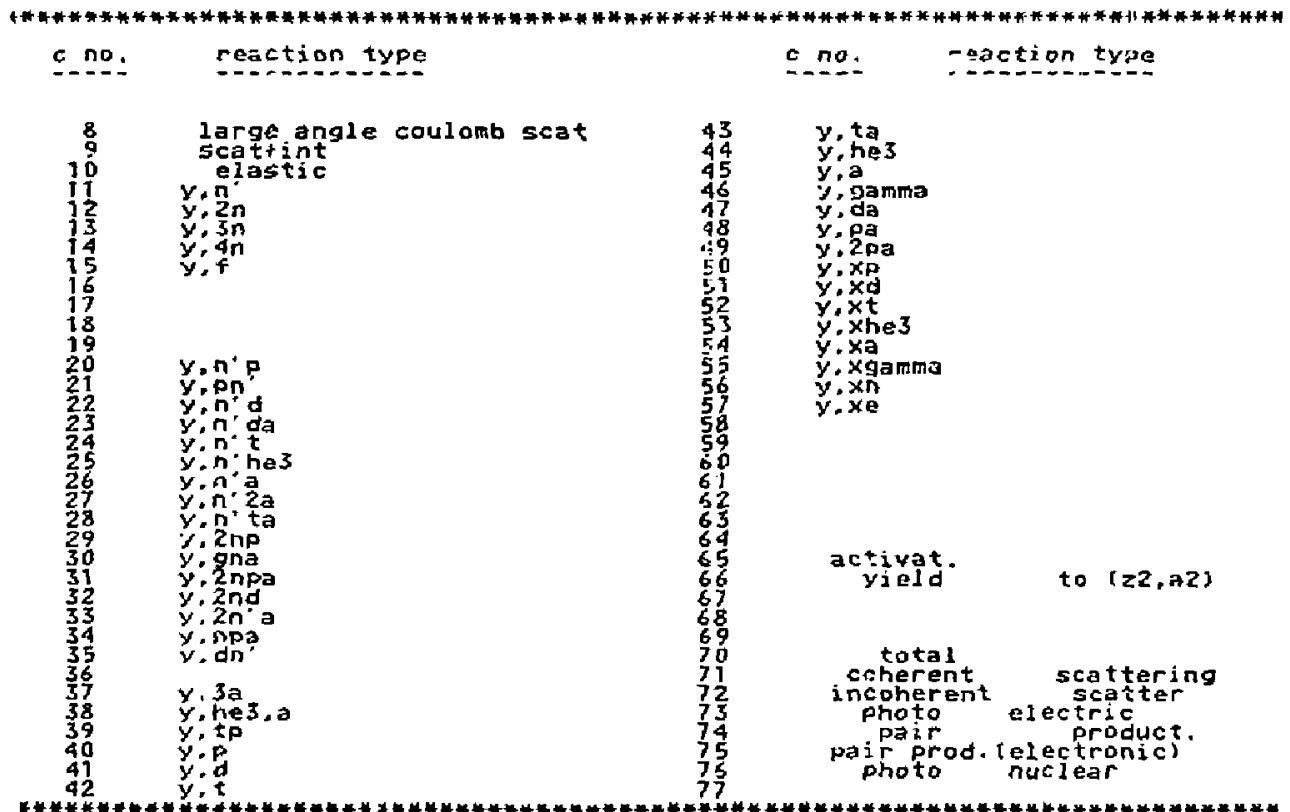


Distribution 1ist.

TID (15 copies)

M. H. Mac Gregor, I-321

External distribucton:

Leona Stewart

Group T-2, Maii Stop B243

Los Alamos National Laboratory

Los A].amos, NM 87545

Rand Me Nally

103 Norman lane

Oak Ridge, Tennessee 37830

B.H. Barschall

University of Wisconsin

1150 University Avenue

Madison, WL 53706

Edward T. Cheng

CA Technologies

P.0. Box B5608

San Dlego, CA 92138

C. Fu

Engtneering Physics Division B1dg. 6010

Oak Ridge National Laboratory

P.O. Box $X$

Oak RIdge, TN 37831

Mohamed Y.A. Gohar

Applied Physics Division

Bldg. 205

Argonne National Laboratory

Argonne, IL 60439

Larry Greenwoö

Fusion Power Program

B1dg. 205

Argonne National Labotatory

Argonne, IL 60439

Robert Haight

MS-0406

Los Alamos Mational Zaboratory

Los Alamos, MM 87545
Glenn Knol1

Bniversity of Mich Igan

119 Cooley Lab, North Campus

Ann. Arbor, Mr 48109

Harold Krox

Accelexator Laboratory

Ohio University

Athens, Ohio $\$ 7701$

Raymond 0. Lane

Acceleracor Laboratory

Ohio University

Athens, Ohio 45701

Duane Larson

EngIteering Physics Division

Oak Ridge National Laboratory

P.O. Box $X$

Oak RIdge, TN 37830

Brad Micklich

Untversity of 2211 noty--Urbana

$103 \mathrm{~s}$. Goodwtn

Urbana, IL 61820

E. Ritter

DOE/Chlcago Operacions office

Argonne National Laboratory

Argonne, II. 60439

Alan Smith

Applied Physics

Bldg. 316

Argonne National Laboratory

Argonne, IL. 60439

Don Smith

Applfed Physics

Bldg. 316

Argonne Nattonal Laboratory

Argonne, It 60439 


\section{Oren Wasson}

Cntr. Rad. Rsch.

National Bureau of Standards

Gatthersburg, MD 20899

\section{Stanley Whetstone}

ER-23/GTN

Division of Nuclear Physics

U.S. Department of Energy

Washington, D.C. 20545

Phil Young

Group $T-2$

Mail Stog B243

Los Alamos Naclonal Laboratory

Los Alamos, NM 87545

Mohmoud Youssef

6288 Boelter Hal 1 , SEAS

UCLA

Los AngeIes, CA 90024

This document was frepared as an acrount af wark spoasored by an agency of the Linited States Govemment. Neither the United States Government nor the liniversity of California nor any of their emplovees, makes any warranty, express or imptied, or assumes any legat liability or responsibility for the accuracy, completeness, or usefulness of eny informution, ap paratus, producl. or process dicclosud, ar represents thu its tse would not inftinge pritately owned rights. Helevence herein to any specific commercial producls, process. ur werviec

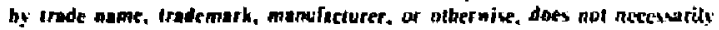
constitutc or imply its endorsement, recommendation, or fectoring by lhe l'nited Sates fionerament ur the I nitersily of California. The siens and opiaimis of zuthmrs puprosed herein do not gecterarily ware or teflere tbone of the I nited

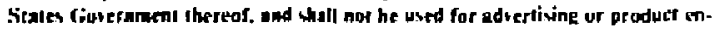
dorsment parpurs, 